\title{
Anesthesia treatment in cases of infant epiglottis cyst emergency extirpation operations
}

\author{
Q. Xu, J.P. Xu and L.Z. Wang \\ JiaXing Maternity and Child Health Care Hospital, JiaXing, ZheJiang, China \\ Corresponding author: Q. Xu \\ E-mail: xuquncn@163.com
}

Genet. Mol. Res. 13 (2): 4523-4528 (2014)

Received May 15, 2013

Accepted October 4, 2013

Published June 17, 2014

DOI http://dx.doi.org/10.4238/2014.June.17.4

ABSTRACT. Congenital epiglottic cyst is a rare embryonic disease. As a congenital laryngeal mucocele, its clinical manifestations include repeated sudden dyspneic respiration and even suffocation accompanied by laryngeal stridor after birth. During food intake, bucking and vomiting is a key feature. Delay in diagnosis and treatment of the disease affects growth and the development of neonatorum leading to suffocation and death. This study was designed to investigate the safety of anesthesia in infants with congenital epiglottic cyst during operation to reduce the occurrence of its complications. The treatment of operations on 12 infants with congenital epiglottic cysts were retrospectively analyzed. Twelve cases of infants with epiglottic cysts received emergency enucleation. Owing to adequate preanesthetic preparation, cystectomies were successfully performed with microwave cauterization under suspension laryngoscopy. None of the 12 patients showed apparent suffocation during anesthesia, the surgical results were good, and after 6 months to 1 year of follow-up, the disease had not recurred. Because of the acute onset of the disease and its severe symptoms and complications, attention should be paid to improve preoperative preparation. Careful selection of proper anesthesia is the 
key to achieving a successful operation.

Key words: Infant; Epiglottis cyst; Emergency surgery; Anesthesia

\section{INTRODUCTION}

Congenital epiglottic cyst is a rare disease that is accompanied by potential airway obstruction (Lee et al., 2000). Congenital epiglottic cyst is a type of congenital laryngeal mucocele that usually occurs in newborns and infants, and results from the obstruction of the mucus duct. The incidence of this disease in newborns is approximately 2 per 100,000 live births (Pak et al., 1996) and can be easily misdiagnosed as pneumonia, congenital throat wheezing, etc. It originates in the residual notochord of the heterotopic nasopharynx, and is mainly caused by the blockage of the mucous glandular tubes, leading to the accumulation of mucous.

Abererombie first named this disease in 1881; however, the pathology, etiology, and treatment was not described in detail until 1978 (Helinger and Barnes, 1978). The main manifestation is upper respiratory tract obstruction, with approximately $40 \%$ of patients showing symptoms within a few hours after birth and $95 \%$ of patients showing symptoms within 6 months after birth. The severe effect of clinical symptoms and the time of syndrome appearance are associated with the size and location of the cyst.

Although infants with small cysts may not show symptoms, the clinical symptoms of those with large cysts include inhelation, cyanosis, dyspnea, and poor effects of oxygen supplementation and choking rescue. Such patients can easily choke to death if they do not receive a prompt diagnosis and surgical treatment.

Previous studies have reported that laryngeal foreign bodies account for 2 to $12 \%$ of all respiratory tract foreign bodies (Daniilidis, 1997; Banerjee et al., 1988), and can exert great harm. Although the incidence of a glottis or subglottis foreign body is low, these are often more critical than tracheobronchial foreign bodies (Halvorson et al., 1996). Because infants cannot describe their symptoms, epiglottis cysts are often misdiagnosed, which increases the chances of suffocation and death.

Furthermore, such young patients with emergency symptoms present a high risk and difficulty in anesthesia treatment. If the disease cannot be treated promptly, it will endanger the patients' life. From June 2008 to June 2011, our hospital received and cured a total of twelve cases of infant epiglottis cysts, and implemented the infants' epiglottis cyst extirpation operations with the support of a laryngoscope microscope. The surgeries were all satisfactorily completed, and there was no recurrence after follow-up.

\section{MATERIAL AND METHODS}

\section{Clinical data}

Among the 12 cases of infants, five were male and seven were female, ranging in age from 1 day to 11 months; two cases were born in our hospital. After asphyxia problems and emergency tracheal cannula, tumors appeared to be present in the pharynx, which were verified by the ear-nose-throat (ENT) doctor using a direct laryngoscope. The severity of symptoms varied among patients. Patients with mild symptoms showed interruptions while sucking 
milk, choking, coughing, crying, or laryngeal stridor, whereas severe patients showed different degrees of inspiratory or mixed breathing difficulty. Among these cases, there were five cases with lung infections, two cases with congenital throat achondroplasia, six cases with over type II degree breathing difficulty, and one in six cases of the newborns appeared to have type IV degree throat obstructions. Patients were given anti-infection and symptomatic treatment, which did not improve the symptoms whether mild or severe. Direct laryngoscope inspection or throat pharyngeal computed tomography (CT) scans confirmed that the neoplasms were oval-shaped ranging in diameter from 1.5 to $3 \mathrm{~cm}$, with the largest one almost completely blocking the pharynx cavity.

Neonatal respiratory difficulties are primarily caused by lung diseases or intracranial diseases. The etiology examinations of infant patients with obstructive breathing difficulties are often ignored after remission, and some patients are treated with laryngeal stridor (John and Swischuk, 1992).

\section{Anesthesia methods}

Before the surgery, newborn infants were injected with $0.1 \mathrm{mg}$ muscle note atropine, and other infants injected with $0.01 \mathrm{rag} / \mathrm{kg}$. Drinking water is forbidden before the operation. The mucosal surface anesthesia method can be used on newborn infants with no tranquilizer to maintain spontaneous breathing and the choke cough reflex, followed by implementing fresh tracheal intubation. If no severe breathing problem is evident in infants that are approximately one year old, they can be given basic, full surface anesthesia and then intubation. Local surface anesthesia can also be used by first applying lidocaine spray at the infants' throat site, and then carrying on with the induction of general endotracheal intubation. For patients whose cysts are too big to expose the glottis, the pumping fluid capsule must first be punctured. In the case of sticky cysts, the hole is slightly expanded, and then part of the content is sucked out with the attractor before reintubation.

For infants who have no breathing difficulties and small cysts, fast induction intubation can be used. After the success of endotracheal intubation, a few fentanyl, muscle relaxant, inhaled sevoflurane anesthesia may be added to maintain the effect according to age. For infants who have smaller cysts, less breathing difficulty, and less local damage, the endotracheal tube can be pulled out after the surgery. However, before pulling out the endotracheal tube, the intubation instruments should be well prepared. If there is a risk of upper respiratory tract obstruction after pulling out the endotracheal tube, it will need immediate reintubation. Infants who have large cysts, severe difficulty in breathing, and have serious surgical wounds will need to be taken back to the intensive care unit with the endotracheal tube. Conventionally, they need antibiotics and dexamethasone. Twenty-four hours after the operation, the endotracheal tube needs to be pulled out after sucking out phlegm.

Congenital epiglottic cysts are derived from the embryonic endoderm and mesoderm cells due to pathological development of the ventriculus laryngis or blockage of glandular tubes. Because the capsule wall is very thin and the surgical field is limited, complete stripping is not absolutely necessary in order to avoid damage to normal tissue. Most of the capsule wall should be removed and the basement exposed to avoid the healing of the capsule wall. This can help to prevent recurrence and achieve therapeutic effects (Chu et al., 1994). 


\section{RESULTS}

Five of the twelve cases had large cysts, which needed casing needle biopsy smoke after fluid intubation.

Due to the well-prepared procedure before anesthesia, none of the sick infants suffered from apparent suffocation during the anesthetic process. Twenty-four hours after the operation, the endotracheal tubes were successfully pulled out, and the patients were discharged in one week. Neonatal intensive care unit patients with endotracheal tubes received hand-controlled oxygen masks, and their airways and infusion channels were also closely monitored. An oxygen pillow and portable oxygen sensing device were carried for security (Table 1).

\begin{tabular}{|c|c|c|c|c|c|c|}
\hline & $\begin{array}{c}\text { Size of kystis } \\
>2.5 \mathrm{~cm}\end{array}$ & $\begin{array}{c}\text { Intubate after drawing } \\
\text { hydatid fluid }\end{array}$ & Dyspnea & Suffocation & $\begin{array}{c}\text { Immediate } \\
\text { extubate }\end{array}$ & Delayed extubate \\
\hline Case & 5 & 5 & 6 & 0 & 8 & 4 \\
\hline
\end{tabular}

\section{DISCUSSION}

Epiglottic cyst is the common disease of otorhinolaryngology. DeSanto et al. (1970) reported that epiglottic cysts accounted for about $52 \%$ of all laryngeal cysts. Due to the small airways of newborns and infants, epiglottis cysts can often cause dyspneic respiration, laryngeal stridor, or dysphagia. Congenital epiglottic cysts are rare and their cause is unknown, although they originate from the obstruction of the submucosal gland duct and the expansion of the secondary glandular organ. The pathological examination results of the two cases of newborn infants with epiglottis cyst were "epidermal inclusion cyst", and were considered as congenital epiglottis cysts. Some researchers have divided congenital throat cysts into two types, in which congenital epiglottis cyst belongs to type I (Forte et al., 2004). Although they belong to the benign lesions category, congenital epiglottis cysts can easily cause breathing difficulty, even leading to death from suffocation. Patients can show clinical symptoms immediately after birth, and the severity degree is related to the size of the cyst. Generally, amniotic fluid and secretions blocking the breathing passage should be cleared so that the choking risk can be quickly dissolved. One such case was observed in the present study: after cesarean section, the newborn baby was in serious suffocation, and the recovery effect was not apparent causing significant breathing difficulty. The infant's face and whole body appeared purple with faint voice, flat former anterior fontanelle, obvious three concave breath symptoms, and flat thoracic symmetry. A gray cystic neoplasm and epiglottis fusion were discovered by direct microlaryngoscopy observation, and the cystic neoplasm was put aside immediately and the endotracheal tube was inserted. For infants with larger cysts and a partially exposed glottis, laryngeal throat spray surface anesthesia was used by direct laryngoscope, followed by case needle biopsy, and the liquid was extracted after withdrawing from the needle core part. For patients with sticky cysts, we first punctured the cyst and then slightly expanded the hole to suck out part of the contents with the attractor before reintubation.

Analysis on these twelve cases of sick infants showed that the main clinical symptoms of infant epiglottis cysts are: throat wheezing (parents were told that the sick infants may have phlegm in their throats and it is was quite apparent when in supine sleeping), which would ap- 
pear immediately after birth or within a few days to a few months following birth, interruption during milk sucking or cough choking, vague crying, breathing difficulties are easily influenced by posture, and inspiratory breathing problems. In some severe cases, obvious breathing problems are apparent such as inspiratory loops for three concave, and sword and rib clearance sag under the axon is more apparent for inspiratory breathing. Therefore, clinical histories should be carefully reviewed when presented with suffocating infants who have been given oxygen recovery but the effect is not apparent. If the symptoms described above are present, the infant should be considered to have an epiglottis cyst.

The stimulus of pediatric laryngofiberscopy examination is low, and it is fundamentally non-invasive. It is easy to operate for skilled operators, and allows complete observation of the throat to facilitate an accurate and timely diagnosis (Liu et al., 1999), thereby leading to timely surgery. However, the clinical symptoms of adult patients suffering from epiglottis cysts are relatively minor, mainly including foreign body sensation in the throat and a sore throat (Nishimura et al., 2008). As the cyst gradually grows, the sensation of pharyngeal foreign body obstruction increases, and huge cysts can ultimately cause dysphagia (Pradeep and Sohail, 2007) and even breathing difficulties (Fang et al., 2002). Epiglottic cyst patients were more prone to acute epiglottitis and dyspneic respiration, which corresponds to previous reports (Yoon et al., 2010). Epiglottic cyst resection is recommended after controlling inflammation to reduce its recurrence. The clinically diagnosed epiglottic cyst should be removed, and to improve symptoms, acute epiglottitis incidence needs to be prevented at the same time (Koga et al., 2004). The simple cyst will be removed by laryngoscope, which is prone to bleeding and damaging the epiglottis mucosa, resulting in obvious local edema. Relapse is common, and physical factors are treated as an effective means to prevent the recurrence of cysts in addition to postoperative laser, microwave, radio frequency (Li et al., 1998), and other means to deal with bleeding and to prevent relapse. At present, the joint use of endoscopy, microscopy, television monitoring system, and minimally invasive instrument surgery has become a trend of minimally invasive surgery of the throat (Kantor et al., 2006).

Anesthesia processing is difficult in treating infants with epiglottis cysts because the cyst is likely to block the chamber and to influence the subglottic exposure, which will lead to difficult intubation. Respiratory inhibition thus occurs easily, resulting in a substantial lack of oxygen that can threaten the patient's life. In such cases, timely use of a manual control mask to achieve positive pressure ventilation and manual extrusion of the chest will help the patient breathe. This can also save valuable time for further processing treatment. Therefore, the anesthesia treatment for infants with epiglottis cysts is very specific and requires a high degree of anesthesia intubation skills. Spontaneous breathing must be maintained while supplying the patients with oxygen and improving their blood oxygen saturation after basic anesthesia. A conventional induction method or muscle loose agent must never be used, which could cause respiratory inhibition. The operation area is in the same airway; therefore, if there is a cyst in the life channel, the patient's safety should be ensured while successfully excising the cyst with the cooperation of emergency medical personnel.

Our hospital is specialized in excising infants' epiglottis cysts with a laryngoscope. The basis of such operations is the use of a self-designed laryngoscope, an electrical attracting hook, a gripping clamp, and other operation equipment. Overall, however, the use of an accurate and proper anesthesia method is the key to successful treatment. Success can only be achieved through the cooperation and hard work of all medical personnel involved. 


\section{REFERENCES}

Banerjee A, Rao KS, Khanna SK, Narayanan PS, et al. (1988). Laryngo-tracheo-bronchial foreign bodies in children. $J$. Laryngol. Otol. 102: 1029-1032.

Chu L, Gussack GS, Orr JB and Hood D (1994). Neonatal laryngoceles. A cause for airway obstruction. Arch. Otolaryngol. Head Neck Surg. 120: 454-458.

Daniilidis J, Symeonidis B, Triaridis K and Kouloulas A (1977). Foreign body in the airways: a review of 90 cases. Arch. Otolaryngol. 103: 570-573.

DeSanto LW, Devine KD and Weiland LH (1970). Cysts of the larynx - classification. Laryngoscope 80: 145-176.

Fang TJ, Cheng KS and Li HY (2002). A huge epiglottic cyst causing airway obstruction in an adult. Chang Gung. Med. J. 25: 275-278.

Forte V, Fuoco G and James A (2004). A new classification system for congenital laryngeal cysts. Laryngoscope 114: 1123-1127.

Halvorson DJ, Merritt RM, Mann C and Porubsky ES (1996). Management of subglottic foreign bodies. Ann. Otol. Rhinol. Laryngol. 105: 541-544.

Helinger LD and Barnes DK (1978). Im'yngocele and saclar cysts. Rhinol. Laryngol 87: 475-485.

John SD and Swischuk LE (1992). Stridor and upper airway obstruction in infants and children. Radiographics 12: 625643.

Kantor E, Berci G and Hagiike M (2006). Operating videoscope for microlaryngeal surgery. Surg. Endosc. 20 (Suppl 2): S484-S487.

Koga T, Shimoda O and Terasaki H (2004). Tracheal intubation using a guide wire for a 5-month-old baby with epiglottic cyst. Masui 53: 79-81.

Lee WS, Tsai CS, Lin CH, Lee CC, et al. (2000). Airway obstruction caused by a congenital epiglottic cyst. Int. J. Pediatr. Otorhinolaryngol. 53: 229-233.

Li KK, Powell NB, Riley RW, Troell RJ, et al. (1998). Radiofrequency volumetric tissue reduction for treatment of turbinate hypertrophy: a pilot study. Otolaryngol. Head Neck Surg. 119: 569-573.

Liu HC, Lee KS, Hsu CH and Hung HY (1999). Neonatal vallecular cyst: report of eleven cases. Changgeng. Yi Xue Za Zhi. 22: 615-620.

Nishimura B, Tabuchi K, Aoyagi Y, Tobita T, et al. (2008). Epiglottic cyst in an infant. Auris Nasus Larynx 35: 282-284.

Pak MW, Woo JK and van Hasselt CA (1996). Congenital laryngeal cysts: current approach to management. J. Laryngol. Otol. 110: 854-856.

Pradeep S and Sohail AM (2007). A new approach for the treatment of large epiglottic cysts using nasoendoscopes. Kuwait Med. J. 39: 59-61.

Yoon TM, Choi JO, Lim SC and Lee JK (2010). The incidence of epiglottic cysts in a cohort of adults with acute epiglottitis. Clin. Otolaryngol. 35: 18-24. 